\title{
Roboterassistierte Prostatektomie - eine Auslegeordnung
}

\section{Franz Recker}

Prof. Dr. med., Senior Consultant Urologische Klinik und Prostatazentrum Aarau

\section{Da das Thema roboterassistierte laparoskopische Prostatektomie sowohl vom} Swiss Medical Board als auch von den Medien zuletzt aus diversen Sichtweisen behandelt wurde, sei es einem Urologen, der seit 1993 offene radikale Prostatektomien (ORP) als auch seit 2006 roboterassistierte laparoskopische Prostatektomien in hoher Anzahl durchgeführt hat, erlaubt, die verschiedenen Aussagen und die Schwierigkeiten ihrer Herleitung näher zu beleuchten.

Das Swiss Medical Board (SMB) kommt zum Schluss, dass es nur eine bedingte Empfehlung für die roboterassistierte laparoskopische Prostatektomie (RALP) geben kann, dahingehend, dass Patienten kurzfristig nach der Operation profitieren könnten, langfristig aber keine Unterschiede zum offenen Vorgehen bestünden und das Verfahren zudem kostenaufwendiger sei. Die Empfehlungen dieses unabhängigen Gremiums beruhen unter anderem auf vier widersprüchlichen, teils zweifelhaften Metaanalysen [1-4] und im Wesentlichen auf einer Cochrane-Analyse von Ilic [5]. Dort werden v.a. die zwei einzigen prospektiv randomisierten Studien aus dem Jahre 2006 [6] in Italien und 2016 [7] in Australien von insgesamt nur $n=428$ Patienten zugrunde gelegt. Darin sind leider auch noch fälschlicherweise $n=120$ Patienten enthalten, die sich nicht einer RALP, sondern lediglich einer konventionell laparoskopischen Operation unterzogen hatten und somit nicht zur gestellten Frage RALP herangezogen werden dürfen [6]. Es verbleiben somit nur $\mathrm{n}=308$ Patienten aus der australischen Studie von Yaxley [7]. Diese 308 Patienten wurden in den Medien als Schlüsselstudie vielfach zitiert. Dies ist, bei alleine im Jahr 2017 weltweit betriebenen $n=4271$ Da-Vinci-Robotersystemen, eine verschwindend geringe Anzahl und mag die Feststellung erlauben, wie ausgesprochen schwierig es für unabhängige und fachfremde Gremien ist, sinnvolle bzw. wegweisende Empfehlungen für gesundheitspolitische Ausrichtungen zu erstellen, auch wenn sie den Kontakt zu Fachvertretern suchen sollten. Natürlich wird der Ruf nach frühen initialen multizentrischen prospektiv randomisierten Studien zur Klärung der RALP-Rolle teils berechtigterweise laut, man bedenke aber bitte auch, dass ein neues Verfahren sich ebenfalls in der Entwicklung befindet und die Frage des Zeitpunktes einer Vergleichsstudienbetrachtung nicht einfach ist. Nichtsdestotrotz ist das Fehlen zu bemängeln.

\section{Fundamentale interne Qualitätsfehler der Schlüsselstudie}

Die oben genannte «Schlüssel»-Arbeit von Yaxley aus dem Brisbane Hospital [7] findet keine relevanten Unterschiede zwischen der offenen und RALP. Obwohl sie den äusseren Qualitätskriterien einer prospektiv randomisierten Studie entspricht, darf sie jedoch wegen fundamentaler interner Qualitätsfehler nicht zum Vergleich ORP und RALP herangezogen werden.

Punkt 1: Innenansicht der zugrunde liegenden Studienqualität von entscheidender Bedeutung Die beiden Operateure Coughlin (für ORP) und Yaxley (für RALP) brachten unterschiedliche, nicht vergleichbare Operationserfahrungen mit (1500 versus 200 Fälle). Diesbezüglich schreibt dann auch der associated Editor des British Journal of Urology International, D. Murphy, 2016 in einem Kommentar: Die einzig zulässige Schlussfolgerung der Publikation sei: «... a much less experienced surgeon can actually deliver equivalent functional and oncological outcomes to a much experienced surgeon, by adopting a robotic approach...»

\section{Punkt 2: Keine State-of-the-art-Performance} Zudem handelte es sich sowohl im offenen als auch im roboterassistierten Arm um eher «unterdurchschnitt- 
liche» Operateure. Der Blutverlust liegt mit 1,338 bzw. 0,443 l zwar eindeutig zugunsten des Roboterurologen, aber trotzdem gehört selbst dieser mit seiner Qualifikation zu den schlechtesten $15 \%$ eines high volume centers [8]. Sein erhöhter Blutverlust von 0,443 l bedeutete eine zumindest teilweise Unübersichtlichkeit im Operationsfeld, sodass die Ergebnisse der RALP wie Frühkontinenz bzw. die Einjahrespotenz um 15 bzw. $20 \%$ schlechter zu erwarten sind als bei mittelmässig bis sehr guten RALP-Operateuren [8]. Beide Arme der australischen Studie repräsentieren damit nicht den State of the Art der Verfahren und dürfen nicht als repräsentativer Vergleich herangezogen werden.

\section{Punkt 3: Kaschierung der erhöhten Blutverluste beim offenen Verfahren}

Noch gravierender ist aber, dass der unüblich hohe Blutverlust des offenen Vorgehens (1,3 l) durch die intraoperative Benutzung von onkologisch äusserst fragwürdigen Cell Savern kaschiert wird, die das verlorene Blut in den Kreislauf zurückführen. Es resultiert eine gleich niedrige Transfusionsrate von $0 \%$ wie bei der RALP und somit kein Unterschied der Verfahren, was schlechthin eine Irreführung ist. Ein Faktum, dessen Analyse für das SMB schwer zugänglich ist.

\section{Punkt 4: Eingeschränkte Studiendetails}

Ausserdem war die Studie vonseiten der Teilnehmeranzahl und der statistischen Power her nur darauf ausgelegt ( $n=151$ vs. $n=157$ ), die gesundheitsbezogenen Fragen der Quality of life nach 24 Monaten zu beantworten, nicht aber frühere Ergebnisse über Potenz und Kontinenz zu beurteilen. Als Nebenbemerkung sei erwähnt, dass die ORP nach 24 Monaten stat. signifikant mehr biochemische Tumorrezidive auswies als die RALP in einer Analyse von 2018 [9]. Aber auch diese Aussage ist nicht zu verwerten, da keine Informationen zu den Salvage Therapien beider Gruppen vorliegen.

\section{Punkt 5: Fehlende volkswirtschaftliche Betrachtung}

Die Studie kann auch den volkswirtschaftlichen Nutzen der RALP nicht dokumentieren, da die Zeitspanne bis zum Wiedereintritt in den Arbeitsprozess mit 47 bzw. 42 Tagen in beiden Gruppen ähnlich ist. Letztere ist insbesondere für die RALP aussergewöhnlich lang, finden sich doch in der Regel Unterschiede zwischen 13 und 36 Tagen zugunsten der RALP [10, 11]. Gerade der frühere Arbeitseintritt ist bei der Gesamtkostenanalyse volkswirtschaftlich einzubeziehen, was das SMB nicht berücksichtigt hat. Interessanterweise ist er zudem bei Selbständigen kürzer als bei Angestellten.

\section{Ein Verfahren allein macht noch keinen Meister}

Zusammenfassend verzerren die Ergebnisse des selbst vom Cochran-Autor Ilic als "Tertiär Center» eingeschätzten australischen Brisbane Hospitals trotz prospektiv randomisiertem Studiendesign eine Bewertung durch das SMB oder die Medien, da es wegen fehlender state of the art performance der Prostatektomien diese nicht wirklich widerspiegelt.

Es stellt sich die Frage, warum die aussagekräftige grösste prospektive, jedoch nicht randomisierte schwedische LAPPRO-Studie mit 2625 Patienten aus sieben offenen Zentren $(\mathrm{n}=778)$ und sieben Roboterzentren ( $n=1847$ ) vom SMB bei der Erstellung ihres Meinungsbildes nicht mit herangezogen wird [10-12]. Hier ist der Roboter bei Low-grade- und Intermediate-grade-Karzinomen der offenen Methode überlegen punkto frühen und langfristigen Potenzerhalts. Bei aggressiven Tumoren ist der Potenzerhalt jedoch nicht besser, da das Robotervorgehen hier eher mehr Radikalität ermöglicht. Das entscheidendste Fazit der Autoren und damit die intraoperative Fähigkeit der filigranen Roboterchirurgie beschreibend, lautet:

«RALP facilitates easier identification of potency nerves (more successful intrafascial preparation) as well as wider dissection in pT3», was ich als langjährige "intrapersonelle Vergleichsperson» beider Verfahren unterstützen kann. Die RALP lässt eine differenzierte schonendere OPTechnik insbesondere in engen räumlichen Verhältnissen zu. Dies äussert sich in einer verbesserten Potenzrate und zudem in einer möglichen geringeren Anzahl von sekundären «Penisverkürzungen» nach RALP [13]. Zwar macht ein Verfahren alleine noch keinen Meister. Die langfristig erworbene Qualität des Operateurs, ob offen oder roboterchirurgisch erlangt, und nicht sein aktueller Case Load ist der entscheidendere Faktor, aber die Optionen mittels RALP sind differenzierter und in Zukunft leichter zu erweitern.

\section{Unterschiedlich steile Lernkurven}

Last but not least ist ein zusätzlicher Gedanke erlaubt. Die Lernkurve für Auszubildende bei der RALP ist kürzer, da sie beispielsweise als Assistenten einen stetigen Einblick über Monitore in den Operationsablauf haben im Vergleich zur ORP. Sie sind dadurch früher befähigt, in den Eingriff eingeführt zu werden. Nicht zu vergessen ist, dass wir in Zukunft eine grössere Anzahl von ausgebildeten Operateuren benötigen, die im Rahmen des gesellschaftlichen Wandels hin zur Work Life Balance und der meines Erachtens viel zu überregulierten europäischen Arbeitszeitnormen notwendig werden. 
Die RALP hat das Potenzial zur Fortentwicklung z.B. durch virtuelle Bildüberlagerungen etc. Ausserdem sollte der vermehrte Kostenaufwand von ca. 20004000 CHF pro Patient mit der geringeren Rehospitalisationrate und der früheren Arbeitsfähigkeit volkswirtschaftlich gegengerechnet werden. Die grösste Krux ist zurzeit das Monopol des Da-Vinci-Systems (Intuitive Companie), das die Anschaffungs-, Unterhaltsund Instrumentenpreise des Roboters noch diktieren kann. Die Patente werden aber sukzessive auslaufen und neue Systeme stehen in den Startlöchern. Die RALP befähigt schneller zu subtilerer und schonenderer OP Technik. Bei ihrer Beurteilung gehören neben dem Ausschluss von insuffizienten Studien Daten auch gesamtvolkswirtschaftliche Betrachtungen mit einbezogen. Ohne dies zu berücksichtigen ist das Statement des SMB nicht nachzuvollziehen. Die Roboter-assistierte Chirurgie steht nach Meinung des Autors am Anfang ihrer Entwicklung.

\section{Literatur}

1 Ficcara V, Novara G, Ahlering TE, Tewari AK, Montorsi F. Systematic Review and Metaanalysis of Studies Reporting Potency Rates After Robotic-assisted Radical Prostatectomy. Eur Urol. Sept 2012;62:418-30.

Korrespondenz: Prof. Dr. med. Franz Recker Senior Consultant Urologische Klinik und Prostatazentrum CH-5000 Aarau Franz.Recker[at]ksa.ch
2 Cooperberg MR, Ramakrishna NR, Duff SB, Tewari AK. Primary treatments for clinically localised prostate cancer: a comprehensive lifetime cost-utility analysis. BJU International. 2013;111:437-50.

3 Hohwü L, Borre M. A short-term cost-effectiveness study comparing robot-assisted laparoscopic and open retropubic rdical prostatectomy. Journal of Medicine Economics 2011;14,403-9.
4 Ontaria HTA (2017). Robotic Surgical System for radical Prostatectomy: A health technology assessment. Ont Health Technol Assess Ser. Canada; 2017:1-172.

5 Evans S, Allan C, Jung J, Murphy D, Frydenbereg M, Ilic D. Laparoscopic and robotic - assisted versus open radical prostatectomy for the treatment of localized prostate cancer. Cochran Database Syst. Rev. Sept 2017

6 Gazzoni G, Cestari A, Zanoni M. Intra- and peri-operative outcomes comparing radical retropubic and laparoscopic radical prostatectomy: results from a prospective randomized single - surgeon study. Eur Urol. 2006;50:98:104

7 Yaxley JW, Coughlin GD, Chambers SK. Robot-assisted laparoscopic versus open radical retropublic prostatectomy: early outcomes from a randomized controlled phase 3 study. Lancet. Sept 2016;10:1057-66.

8 Preisser F, Pompe RS, Salomon G, Rosenbaum C, Graefen M, Huland H, Tilki D. Impact of the estimated blood loss during prostatectomy on functional outcomes. Urol Oncol. 2019;37:298.

9 Caughlin GD, Yaxley JW, Chambers SK. Robot-assisted laparoscopic versus open retropubic prostatectomy: 24 months outcomes from a randomized controlled study. Lancet. Aug 2018;19:1051-60.

10 Wallerstedt L, Stranne J, Tyritzis SI, Carlsson S, Hugosson J, Haglind E. 90 Days readmission after radical prostatectomy - a prospective comparison between robot and open surgery. Scand J Urol Feb 2019;6:1-8.

11 Haglind E, Carlsson S, StranneJ Wallerstest A, Wiklund P and the LAPPRO steering committee. Urinary incontinence and Erectyle Dysfunction After Robotic Verus Open Radical Prostatectomy: A Prospective, Controlled, Nonrandomized Trial. Eur Urol. Aug 2015;68:216-25.

12 Sooriakumaran P, Pini G, Nyberg T, Wiklund PN. Erectyle Function and Oncological Outcomes Following Open Retropubic and Robot assisted Radical Prostatectomy: Results from the LAParoscopic Prostatectomy Robot open Trial. Eur Urol. 2018;73:618-27.

13 Capogrosso P, Ventimiglia E, Cazzangia W, Salonia A. Long term penile morphometric alterations in patients treated robotic versus open radical prostatectomy. Andrology. Jan 2018;136-41.

\section{Replik zum Artikel von Franz Recker}

\section{Expertenrat des Swiss Medical Board und Mitglieder des Assessment-Teams}

Mit Interesse haben wir den Beitrag von Prof. Dr. med. Franz Recker zum Bericht des Swiss Medical Board (SMB) betreffend roboterassistierte Operationsverfahren gelesen [1]. Hiermit möchten wir zu dem Gesagten Stellung nehmen; und zudem eine Frage aufwerfen, die die Beweislast bei der Einführung neuer medizinischer Technologien betrifft.

\section{Zum Inhalt des SMB-Berichts}

Wir wollen darauf hinweisen, dass der SMB-Bericht nicht nur die roboterassistierte laparoskopische Prostatektomie, sondern auch die roboterassistierte lapa- roskopische Hysterektomie behandelt, bei der die Evidenzlage für den Einsatz von Robotern noch schwächer ist [2]. Andere roboterassistierte Eingriffe, wie zum Beispiel die bariatrische Chirurgie, wurden im Bericht nicht erwähnt, da für diese keinerlei randomisierte Studien vorlagen. 


\section{Unsere Antworten im Einzelnen}

Gerne gehen wir im Folgenden auf die vom Autor vorgebrachten Argumente ein.

- Bei den drei zitierten Publikationen von Cooperberg, Hohwü und Ontario handelt es sich nicht wie vom Autor geschrieben um Metaanalysen, sondern um gesundheitsökonomische Studien.

- Die teilweise berechtigte Kritik des Autors an der Studie von Yaxley wurde im SMB Assessment Report diskutiert [3].

- Der Autor spricht von einem hohen Blutverlust bei der offenen Prostatektomie (1,3 Liter), der durch die Benutzung von "onkologisch fragwürdigen Cell Savern [...] kaschiert wird». Aus der im SMBBericht analysierten Literatur sowie den darin beschriebenen Outcomes kann leider kein Schluss betreffend die onkologische Fragwürdigkeit oder Häufigkeit der Anwendung von Cell Savern gezogen werden. Urologische Fachexperten vertreten die Meinung, dass vermutlich der Chirurg der relevantere Faktor ist, verglichen zum Instrument.

- Der Autor erwähnt, dass der Wiedereintritt in den Arbeitsprozess bei der roboterassistierten laparoskopischen Prostatektomie «13-36 Tage früher» erfolgt. Die Analysen im SMB-Bericht basieren auf Daten von publizierten randomisierten Studien; für die von Herrn Prof. Dr. med. Recker erwähnten Daten fehlt leider die entsprechende Literaturreferenz.

- Der Autor hält fest, dass die seines Erachtens aussagekräftigste LAPPRO-Studie nicht in unsere Analyse einbezogen wurde. Dies ist richtig, haben wir uns doch ausschliesslich auf Daten von randomisierten Studien bezogen. Ob bei der Abwägung von Effizienz und Risiko einer Behandlung Daten von unkontrollierten, aber qualitativ hochwertigen Beobachtungsstudien ergänzend zu randomisierten klinischen Studien herangezogen werden sollen, ist tatsächlich immer wieder ein Thema, das auch im Expertenrat des SMB intensiv diskutiert wird, besteht doch bei nicht-randomisierten Studien immer das Risiko für das Auftreten von systematischen Störfaktoren ("confounding factors» und «biases»), für die es teilweise schwierig bzw. unmöglich ist, eine statistische Korrektur durchzuführen. Die LAPPRO-Studie wird allerdings im Anhang des Berichtes des SMB-AssessmentTeams erwähnt (Seiten 185-186).

Korrespondenz:

Prof. Christoph A. Meier Swiss Medical Board

Stampfenbachstrasse 30

Postfach

CH-8090 Zürich

info[at]swissmedicalboard.ch nen" explizit angesprochen, dass keine ausreichende Datenbasis bestand, um solche Aspekte quantitativ zu berücksichtigen, umso mehr als diese von vielen verschiedenen Faktoren abhängen, wie z.B. Schulung, Zahl der assistierten Eingriffe und Qualitätsmonitoring.

\section{Eine andere Frage steht im Vordergrund}

Viel wichtiger als die Diskussion der oben erwähnten Punkte ist allerdings die grundlegende Frage, wo die Evidenzlast bei der Einführung neuer medizinischer Technologien liegen soll. Aktuell ist es, wie im vorliegenden Beispiel, häufig so, dass HTA-Gremien, Krankenversicherer, Regulatoren und alle anderen potenziell betroffenen Interessengruppen dazu aufgefordert sind, zu beweisen, dass die neue Technologie der herkömmlichen nicht unterlegen sei - und dies führt genau zu den oben erwähnten Debatten. Viel sinnvoller wäre es jedoch, wenn neue Technologien vor oder während ihrer Einführung von überzeugenden klinischen Studien begleitet würden, die die Evidenzlast bei den Innovatoren ansiedeln, wie dies ja zum Beispiel bei der Medikamentenentwicklung seit Langem der Fall ist. Da dem aber in der Praxis bei neuen medizinischen Technologien (noch) nicht so ist, bestehen meistens keine Datengrundlagen für solide evidenzbasierte Entscheide.

\section{Fazit}

Es sollte ernsthaft erwogen werden, dass medizintechnische Innovationen, soweit sie nicht schon bei ihrer Einführung medizinisch und gesundheitsökonomisch evidenzbasiert dokumentiert sind, spätestens ab dem Zeitpunkt der klinischen Einführung in prospektiven Registern mit vordefinierten Endpunkten begleitet werden. Somit könnten nach einigen Jahren, falls die nötigen randomisierten Studien immer noch nicht vorliegen, medizinisch und ökonomisch begründete Entscheide betreffend Wirksamkeit, Wirtschaftlichkeit und Risiko solcher Innovationen zumindest basierend auf gut dokumentierten, unabhängigen Registerdaten getroffen werden.

\footnotetext{
Literatur

1 Recker F. Roboterassistierte Prostatektomie - eine Auslegeordnung. Schweiz Ärzteztg. 2019;100(31-32):1037-39.

2 https://www.swissmedicalboard.ch/fileadmin/public/news/2018/ Summary_appraisal_EtD_hysterectomy_def.pdf

3 https://www.swissmedicalboard.ch/fileadmin/public/news/2018/ assessment_report_smb_robot-assisted_surgery_long_2018.pdf, Seite 118 .
} 